\title{
Science indicators and science patterns in Europe
}

\author{
J.A.S. Almeida, A.A.C.C. Pais, S.J. Formosinho* \\ Chemistry Department, University of Coimbra, Rua Larga, 3004-535 Coimbra, Portugal
}

\section{A R T I C L E I N F O}

\section{Article history:}

Received 29 October 2008

Received in revised form 31 December 2008

Accepted 7 January 2009

\section{Keywords:}

European science

Scientific productivity

Essential science indicators

Web of science

\begin{abstract}
A B S T R A C T
In this communication we perform an analysis of European science, investigating the way countries are joined in clusters according to their similarity. An extremely clear pattern arises, suggesting that geographical and cultural factors strongly influence the scientific fabric of these countries. Although it is seen that one of the major factors behind Science in Europe is, apparently, geographical proximity, bilateral cooperation between countries cannot fully account for the respective similarity. Long-term policies, planning and investment are also visible in the results.
\end{abstract}

(c) 2009 Elsevier Ltd. All rights reserved.

\section{Introduction}

The access to bibliographic and citation databases is changing the way scientific productivity is evaluated (Adam, 2002; Coccia, 2008; Cronin, 2001; Csajbók, Berhidi, Vasas, \& Schubert, 2007; Moed, De Bruin, \& van Leeuwen, 1995; Thelwall, 2008; Tian, Wen, \& Hong, 2008; Weingart, 2005). There is, however, a lack of understanding of what can be extracted from the data, and science policies based on this information are difficult to design (van Raan, 1998, 2005).

The understanding of how research is structured around the world, and what are the main national and international characteristics and patterns, is becoming increasingly important in order to support more global policies (Glänzel, 2007; King, 2004; Kostoff et al., 2007; Kozlowski, Radosevic, \& Ircha, 1999). The use of scientometric indicators in research evaluation emerged in the 1960s and 1970s (Leydesdorff, 2005) after the Garfield proposal of a global scientific citation index (Garfield, 1955), and is widely in use today due to easy access to the relevant databases. Individual institutions and researchers are now classified on the basis of scientific productivity, usually established using the number of papers, the impact factors for the journals and the number of citations (see, e.g., Bishop, Gillet, Holliday, \& Willet, 2003; Braun, 1999; Hirsch, 2005; Schreiber, 2008; Winkler, 2007). However, as pointed out by some authors (Braun, Dióspatonyi, Zádor, \& Zsindely, 2007), attempts at ranking institutions and scientists have sometimes produced disparate and inappropriate results. In fact, the difficulty is to clearly understand what good science is and to know if data reflects quality. It becomes even more difficult when trying to understand the trends and underlying variables that govern the scientific fabric of a country or groups of countries (Horta \& Veloso, 2007; Ortega \& Aguillo, 2008). Moreover, as previously stated, "failure to relate interpretations of specific measures to the historical development of science can lead to errors in assessing past investments and in prioritizing future investments" (Feller \& Gamota, 2007).

As a contribution to understand how science is organised in Europe, we analyse the scientific productivity of various countries in the last 10 years. Our main purpose is to define indicators that can be used to characterise the structure of science of a country, and use it to extract inter-country relationships and patterns. For this, we select a set of variables

\footnotetext{
* Corresponding author. Tel.: +351 239854466; fax: +351239827703.

E-mail address: sformosinho@qui.uc.pt (S.J. Formosinho).
} 
related with the scientific production in each area, and inspect the pattern that arises resorting to hierarchical cluster (HCA) and principal component (PCA) analyses. We also intend to discuss other important aspects for the evaluation of science, including the quantity/quality assessment.

The present study comprise the EU-15 countries, excluding Luxembourg, plus the recent EU members Czech Republic, Hungary, Poland and Slovakia. Also included are the non-EU countries Switzerland, Norway, Russia and Ukraine, and the candidate Turkey. Exclusions among European countries are based on low dimension in terms of citations, as detailed below. According to the databases, UK is treated as four separate territories: England, Northern Ireland, Scotland and Wales.

\section{Sources and methods}

Publication and citation data were taken from Essential Science Indicators ${ }^{S M}$ and Web of Science ${ }^{\circledR}$, part of ISI Web of Knowledge $^{S M}$ (ISI Web of Knowledge, URL accessed in November 2007). Data from the Essential Science Indicators ${ }^{S M}$, ESI, corresponds to November 2007. Covering a multidisciplinary selection of more than 11,000 journals, ESI is based on a 10-year period collection of data (publication and citation counts) organised in rankings of researchers, institutions, countries/territories and journals. Other details concerning the specific procedures relevant for the database can be found in reference (Essential Science Indicators, URL accessed in November 2007). Some data taken from Web of Science ${ }^{\circledR}$, WoS, corresponding to the year 2006, was also used to perform the analyses. WoS consists of various citation databases containing bibliographic information gathered from thousands of scholarly journals in all areas of research (Web of Science, URL accessed in November 2007).

In what follows we use hierarchical cluster analysis, HCA, for which a recent review can be found in reference Almeida, Barbosa, Pais, \& Formosinho (2007). Briefly, scattered objects are joined in decreasing order of similarity. The first (and most significant group) is made up from the two most similar countries. The subsequent steps test not only the distance between countries, but also between a country and a group, or two groups already formed. To define the latter two distances, one must establish a linkage scheme. One of the usual linkage approaches is average-linkage, in which the distance between objects or groups is based on averaging over the members of the clusters. This procedure will be followed in the present work, but other linkage approaches, including Ward's method referred as one of the most generally useful hierarchic procedure (Willett, 1988), were also tested. In Ward's method, the selection of two clusters that merge in one is governed by the minimisation of the increase in the error sum of squares, calculated as the deviations from the centroids of the clusters, before merging, and the new group, after merging.

Principal component analysis (PCA) is an appropriate technique for extracting underlying factors from a multivariate dataset. Based on an orthogonal linear transformation, PCA defines a lower dimensionality system, such that the highest variance of the data comes projected on the first principal component (PC1), the second largest on the second coordinate (PC2), and so on (Brereton, 2003). PCA is usually performed after centering the data, and is based on the solution of an eigenvalue problem, either based on the correlation or variance/covariance matrix of the original variables. In this work latter is used.

Varimax searches for a rotation (i.e., a linear combination) of the PCA axis (Abdi, 2003), such that the variance of the loadings, i.e., the weights of the original variables, are maximized. It facilitates the inspection of groups of variables present in the axis considered, allowing to rationalise the PCA results and determine the underlying factors.

\section{Results and discussion}

\subsection{Quality vs. quantity}

It is extremely relevant, when one tries to characterise the scientific productivity, to establish the distinction between quality and quantity. In fact, the interpretation retrieved from most of the analyses to data presented in ESI reflects quantity or dimension. Institutions and countries/territories are listed if they are found, respectively, within the top 1 and $50 \%$ in terms of number of citations (Essential Science Indicators, URL accessed in November 2007). However, as shown in Fig. 1, there is a clear correlation $(r>95 \%)$ showing that countries/territories from which a large number of papers is published also receive a significant number of citations, suggesting that some mixture between quantity and quality may occur.

Focusing on institutional analysis, this means that institutions with large staffs are more likely to produce a large number of papers. Furthermore, the number of papers correlates (with a correlation coefficient of 90\%) with the 2006 gross domestic product (GDP) (International Monetary Fund, URL accessed in November 2007), once again emphasising the quantitative nature of the indicator (see also King, 2004 and May, 1998 in which a more direct relationship is established with the GDP per person and R\&D investment, respectively). This result is depicted in Fig. 2.

In summary, larger countries or larger institutions publish more papers, and these receive a proportionally higher number of citations. In terms of policy, this suggests one trivial rule: institutions within a country should merge, especially in smaller countries, for a better positioning in the rankings. This may not seem to be a very relevant conclusion. However, the prominence of large institutions, although clearly not ruling out the quality factor, may to a significant degree be attributed to the institutional dimension (number of researchers, resources and funding). This is consistent with recent observations from several quality and efficiency criteria, showing that the United States are outperforming Europe in terms of science (Andras \& Charlton, 2004). It is pointed out that one clear difference between Europe and 


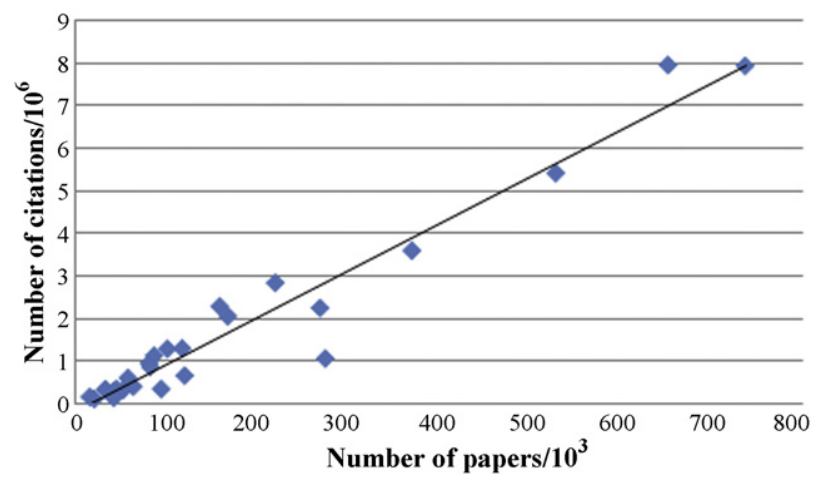

Fig. 1. Representation of the number of papers against the number of citations for the set of European countries under study.

United States is science funding, which is proportionately higher in the United States and concentrated in relatively fewer institutions.

\subsection{Hierarchical cluster and principal component analyses}

We now refine the analysis, using vectors of variables defining the European countries/territories. For each field included in ESI, organised for example in alphabetical order, the number of citations divided by the total for the country dictates each component of the vector. Using this set of variables, we have built a similarity matrix for the European countries/territories with over 100,000 citations in the period considered. Note that the 26 countries/territories under study represent more than $95 \%$ of the whole papers and citations in Europe. The similarity matrix is built using Euclidean distance, i.e., the most similar countries/regions are those for which this distance is smaller. Similar countries have, thus, a matching profile in what concerns the relative impact of the different areas, as measured from the number of citations.

Representing each object (country/territory) as a point in the hyperspace of scientific areas, through data extracted from ESI, allows us to carry out the HCA that result in the dendrogram presented in Fig. 3. This is a pictorial representation of the data structure, indicating the merging objects and the merging distances.

Fig. 4 summarises the closest connections found in the dendrogram. On a first analysis, it is seen that geographical proximity, and its consequences such as language and culture, presents itself as the major factor responsible for the structure depicted in the dendrogram. In fact, with very few exceptions, neighbouring countries are those merged in the most significant groups. The expansion of these groups for larger aggregates also tends to initially include countries that are geographically very close.

The two countries that present most similar patterns are France and Germany, which then gather Switzerland and, after, Spain. France and Germany do not share a common language and had long periods of poor relations and war. However, they have a recent past of intense collaboration in terms of resources and a significant portion of common views within the EU. They present some exchange of population and mutual influence in regions such as the Alsace-Lorraine territory. Below we will detail the degree of scientific cooperation.

Spain represents the single divergence when comparing average linkage with Ward's method, emerging connected to Hungary when resorting to the latter. Belgium and The Netherlands are also in close proximity, as are Denmark and Sweden. They initially form two groups, connected in a subsequent step, and are later joined with the Austria/Italy group. It is interesting that the Denmark/Sweden group tends to merge with Belgium and The Netherlands instead of Finland/Norway.

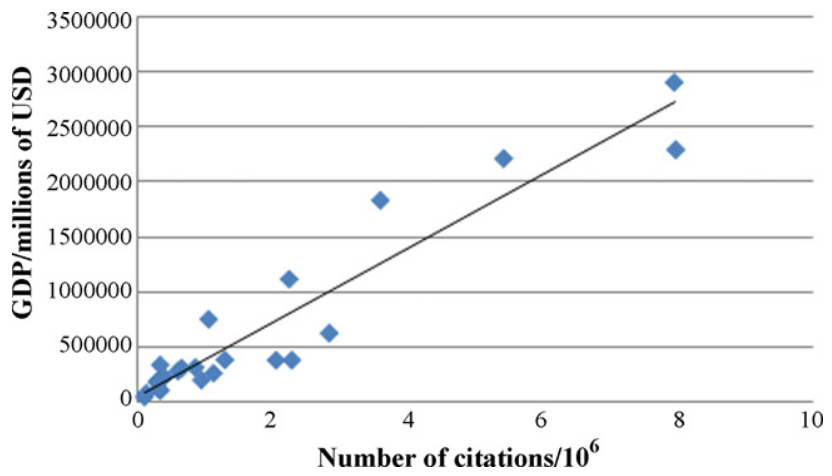

Fig. 2. Representation of the number of citations against the GDP for the set of European countries under study. 


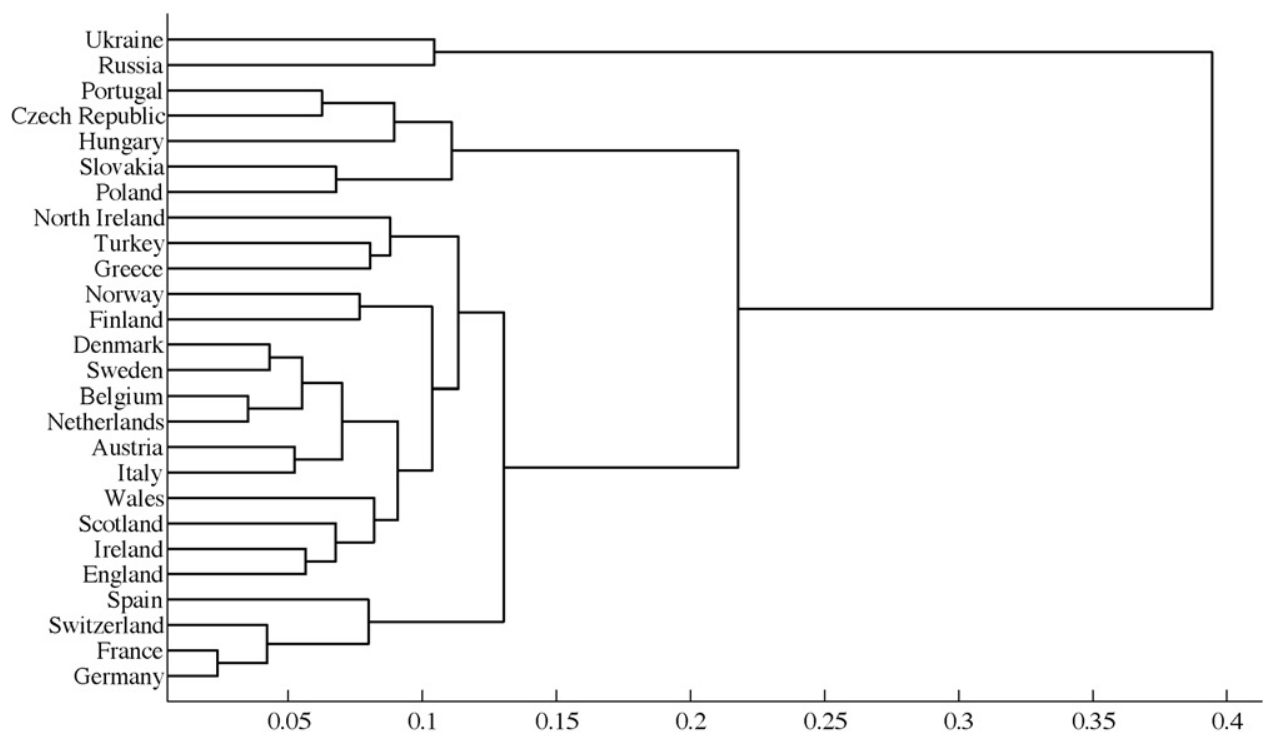

Fig. 3. Similarity among European countries in terms of the distribution of scientific productivity by the different areas. Dendrogram obtained resorting to average-linkage with Euclidean distances, using the number of citations per area as variables defining each country/territory.

This arrangement is hard to extract from development indicators, in which a frequent positioning for these countries is in the order Norway, Sweden, Finland and Denmark (see, e.g., the 2008 update of the UN Human Development Indices, HDI, URL accessed in December 2008). If we inspect closely the respective profiles, it is apparent that Norway has some particular characteristics, such as a higher relative development in Environment \& Ecology and, especially, Geosciences. Finland and Norway present a higher impact in Clinical Medicine than either Sweden, Denmark, Belgium or The Netherlands. On the other hand, Biology \& Biochemistry and Chemistry are more important in the latter.

Common borders, mutual linguistic influence, the historical importance of music are some of the factors that may account for the formation of the Italy/Austria group. The similarity does not result from direct scientific cooperation, as will be shown later.

England joins Ireland first, then Scotland and Wales. Finland and Norway, as Turkey and Greece, also initially form clusters, but the patterns do not match closely. The same happens for Ukraine and Russia: they are not very similar but are closer to each other than to the other European countries. Poland and Slovakia, with a closely related language, are more similar. The former Eastern block is split: Ukraine and Russia isolated, with another group formed by Poland, Slovakia, Hungary and the Czech Republic, i.e., countries that have joined in 2004 the European Union, EU. This latter group also comprises Portugal, in one of the few exceptions in the geographical proximity rule. Portugal is most similar to the Czech Republic, and this group is subsequently joined by Hungary. Probably, the fact that these countries occupy a peripheral position in Europe has conducted to this result. It means that they have been exposed throughout history to external influences, sometimes coincident. As an example they, with Spain, have been an advanced post of European society against Saracen invasions. Also, Portugal, the Czech Republic and Hungary are close in what concerns the HDI (2008) (in 33rd, 35th and 38th position, respectively).

There are other examples showing that not only geographical considerations determine the present pattern: Northern Ireland joins the Turkey/Greece group and is thus surprisingly not present in the geographically close England/Ireland-Scotland/Wales cluster. If one inspects closely the differences between Northern Ireland and England, it is seen that it is located in emerging fields (such as Molecular Biology \& Genetics) which have not attained in the former the relative importance they have in the latter. This may be tentatively ascribed to the social instability found in Northern Ireland until recent years, which may have affected the development of these new areas in relation to those of longer tradition. The region becomes thus closer to Turkey and Greece, but the degree of similarity is low.

In practical terms, the most significant gap found in Fig. 3 corresponds to the separation between the set Russia/Ukraine from the rest of Europe. This seems to arise from the relatively higher productivity achieved in Physics by Russia and Ukraine (respectively, 41 and $36 \%$ of the total for each of these countries). If we go deeper, the remaining countries of the former Eastern block, and Portugal, separate from the other Western and Central countries. Those Eastern countries are strong in Chemistry (17-21\%), but also in Physics (13-24\%) and Clinical Medicine (12-17\%). Clinical Medicine (typically above 30 , systematically above $20 \%$ ) is definitely the area of larger productivity in Central and Western Europe. Biology \& Biochemistry is almost always the fourth most relevant field and, together with Clinical Medicine, Chemistry and Physics, accounts for more than $50 \%$ of all citations in the period under study. Fig. 5 illustrates these observations.

Interestingly, marked evidences of the above structure are found in the PCA result. In fact, only two PC account for more than $95 \%$ of the initial variability. Then, subjecting loads to a Varimax rotation, it is clear that Clinical Medicine, Physics and 


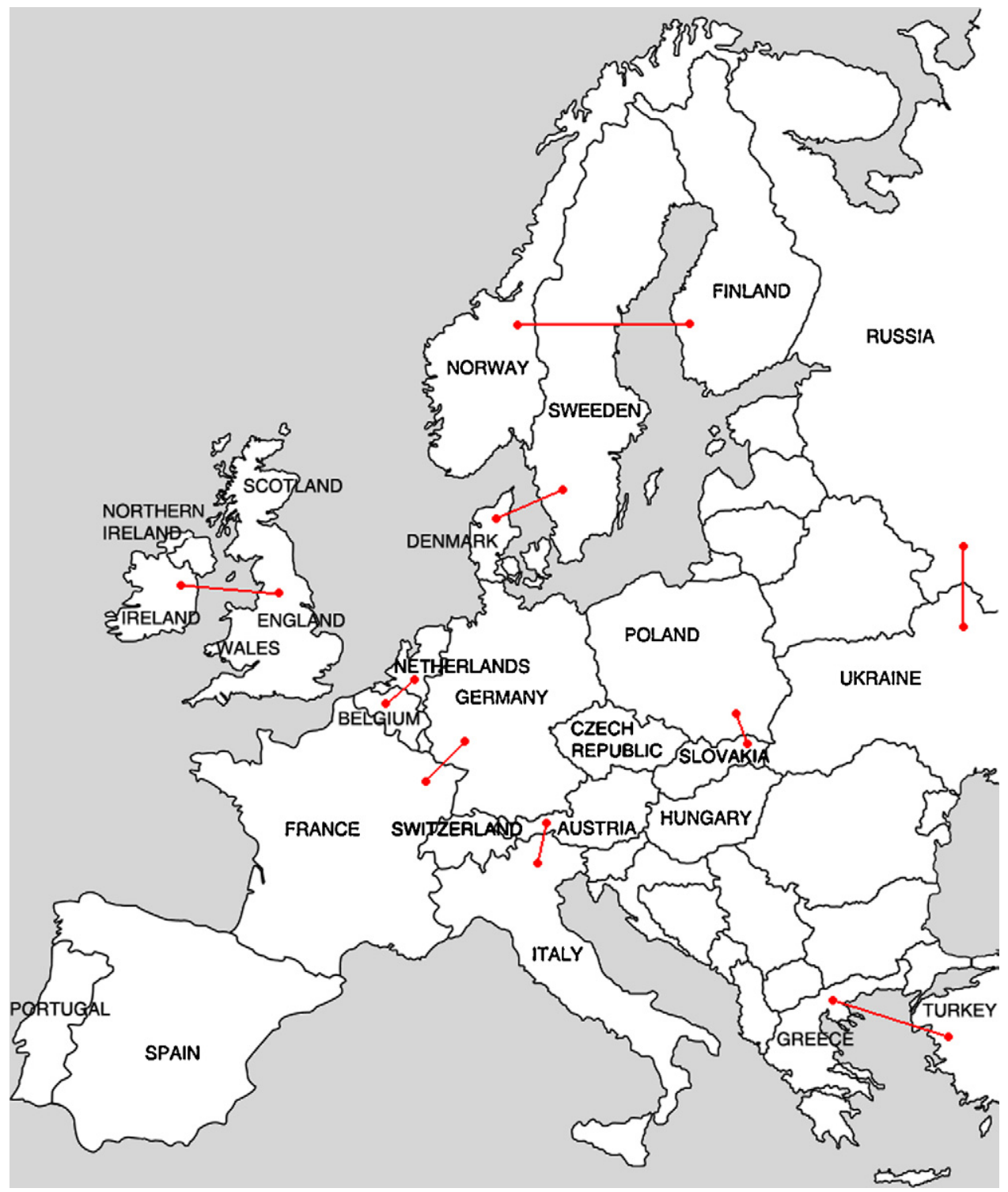

Fig. 4. Representation upon the European map of the closest connections from the dendrogram depicted in Fig. 3.

Chemistry are three important variables in the characterisation of the data, arising separated from the rest of the set of whole variables (Fig. 6).

The geographical pattern and the PCA result also shed some light on the question of the split of science from the humanities, which C. P. Snow addressed in his article (Snow, 1964) titled "The Two Cultures" on 1964, and is a widely accepted view in modern times. Snow attributes this gap to specialization. However, the physical-chemist Michael Polanyi (Polanyi, 1983) reacted against the existence of such a split arguing, in terms of his theory of tacit knowledge, that there is continuity between the natural sciences and the humanities. In his own words: "Tacit assent and intellectual passions, the sharing of an idiom and of a cultural heritage, affiliation to a like-minded community: such are the impulses which shape our vision of things on which we rely for our mastery of things. No intelligence, however, critical or original, can operate outside such a fiduciary framework". When we look at Fig. 6 we also see that the separation between science and the humanities is absent. We additionally note that the overall pattern depicted in Fig. 3 is obtained even after removing the data pertaining to humanities, i.e., Social Sciences (general), Psychiatry/Psychology and Economics \& Business. It should be said, however, that these areas have a relatively low weight in the information extracted from the database. Nevertheless, the present findings seem to support Polanyi's view, and contrast with the ideal of an impersonal knowledge that gains a paradigmatic expression in positivism. 


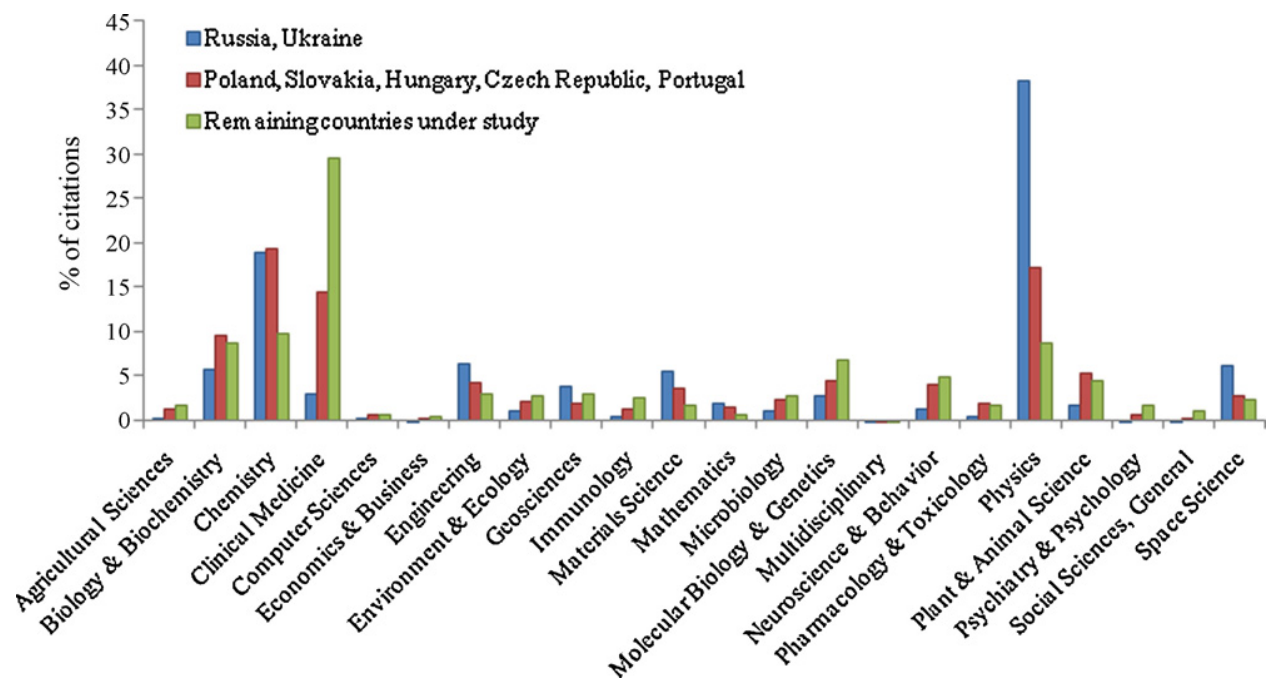

Fig. 5. Scientific profile for the three major groups found in the similarity analysis. Relative importance of the indicated scientific areas based in the number of citations, normalised for each country with the total, and averaged over the countries belonging to each group.

We note that the patterns obtained in the characterisation of Science in Europe are also related with the degree of evolution in terms of scientific productivity (see also May, 1997). Without detailing, we just stress some relevant aspects of this evolution. Looking at the last 10 years, Russia is publishing less today than in $1997(-12 \%)$, and Ukraine is almost not growing (2\%). Most Central Europe countries display a relatively moderate growth (typically below 50\%). Following a clearly increasing trend are Turkey (307\%), Portugal (212\%), Greece (138\%) and Poland (95\%).

One question that deserves further analysis is the degree of cooperation between countries and how it is related with the respective similarity. For this analysis, data from Web of Science ${ }^{\circledR}$ concerning 2006 was used. This analysis was conducted considering just 1 year, instead of the ten used in the pattern analysis, because the number of publications returned by WoS for some countries exceeds the maximum of 100,000 publications allowed by the tool. The USA were also included in the analysis as an external influence. Due to its dimension, the USA may have a significant impact in the Science profile of some European countries. It is interesting to see that most European countries have, in 2006, maintained a degree of cooperation with researchers in the United States superior to that with other countries of the continent. This is true for Germany (13\%), England (13\%), France (12\%), Italy (12\%), Spain (10\%), etc., and, typically, more than $10 \%$ of the scientific papers published in most European countries also include American researchers. However, cooperation with the USA does not emerge as a discriminant factor, since all the countries present a similar fraction of collaboration, $11.85 \pm 2.25$. Resorting again to Fig. 3,

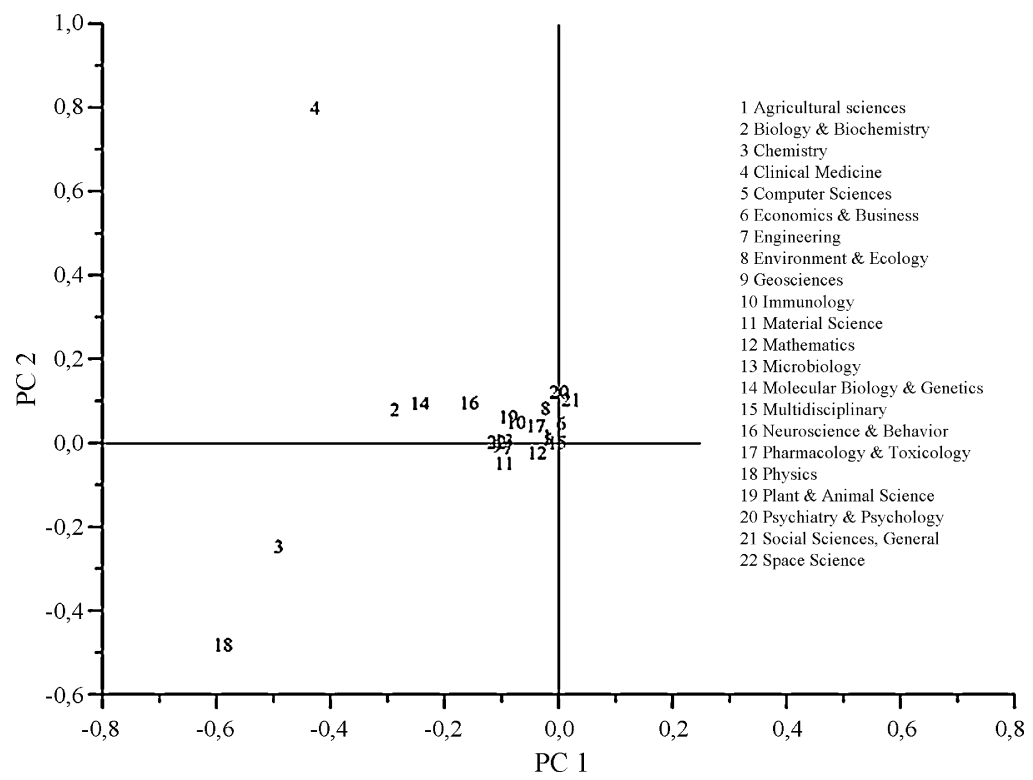

Fig. 6. Representation of the loads on the principal components axes, rotated according to the Varimax method. 
Table 1

Four most important collaborations, defined as the fraction of joint papers to the total of each country, organised in descending order of importance.

\begin{tabular}{|c|c|c|c|c|}
\hline & $1 \mathrm{st}$ & 2nd & $3 r d$ & 4 th \\
\hline ENGLAND & Germany & France & Scotland & Italy \\
\hline GERMANY & England & France & Switzerland & Italy \\
\hline FRANCE & Germany & England & Italy & Spain \\
\hline ITALY & Germany & France & England & Spain \\
\hline NETHERLANDS & Germany & England & France & Belgium \\
\hline SWITZERLAND & Germany & France & England & Italy \\
\hline SPAIN & France & Germany & England & Italy \\
\hline SWEDEN & Germany & England & France & Denmark \\
\hline BELGIUM & France & Netherlands & England & Germany \\
\hline SCOTLAND & England & Germany & France & Italy \\
\hline DENMARK & England & Germany & Sweden & France \\
\hline RUSSIA & Germany & France & England & Italy \\
\hline FINLAND & Sweden & Germany & England & France \\
\hline AUSTRIA & Germany & Italy & England & Switzerland \\
\hline POLAND & Germany & France & England & Italy \\
\hline NORWAY & England & Sweden & Germany & Denmark \\
\hline GREECE & England & Germany & France & Italy \\
\hline HUNGARY & Germany & England & France & Italy \\
\hline TURKEY & Germany & England & Italy & France \\
\hline WALES & England & Germany & Scotland & France \\
\hline IRELAND & England & Germany & France & Italy \\
\hline CZECH REPUBLIC & Germany & France & England & Italy \\
\hline PORTUGAL & Spain & England & France & Germany \\
\hline NORTH IRELAND & England & Scotland & France & Germany \\
\hline UKRAINE & Germany & Russia & Poland & France \\
\hline SLOVAKIA & Czech Republic & Germany & Poland & France \\
\hline
\end{tabular}

we see that France and Germany are very similar and, in fact, from the total of French publications, 7\% are jointly authored with German researchers. In this case, most of the within-Europe French cooperation is thus with neighbouring Germany. However, if we look for example to Italy, which is similar to Austria, only 1\% of the papers involve Austrian researchers, contrasting to a common figure of ca. 7\% with either France, Germany or England. The number of joint publications between Austria and Germany is above three times the corresponding value for Austria and Italy. The same situation is found for other countries, as stressed in Table 1 where for each country we list the four with which collaboration is highest. England, Germany and France are almost always present in this set of collaboration countries, which means that strong countries from a scientific point of view are more attractive in terms of cooperation. In some cases, at a secondary level, it is possible to find some correlation with the structure previously found. However, the degree of joint effort does not seem to explain the whole pattern. A more complete analysis for the period 2000-2002 can be found in reference (Schubert \& Glänzel, 2006). Some aspects concerning geographical factors have also been discussed in the same reference.

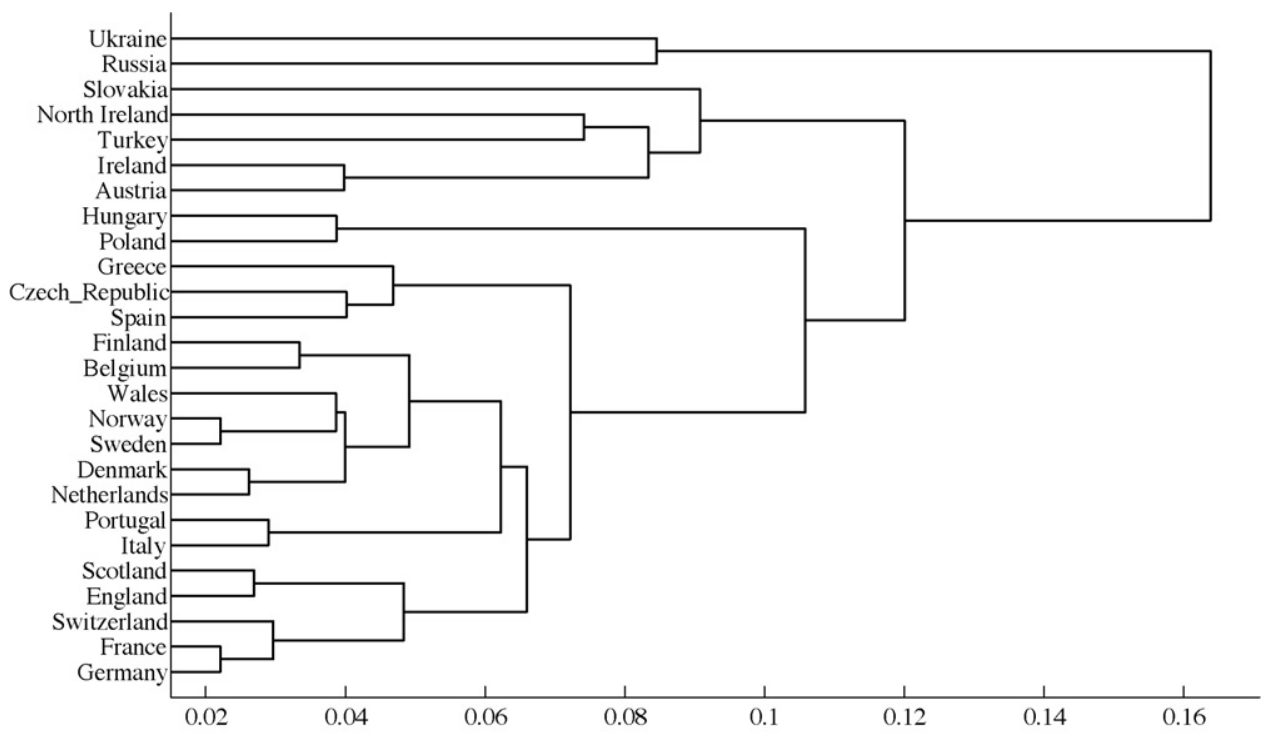

Fig. 7. Dendrogram obtained resorting to Ward's method with Euclidean distances, using the number of citations per paper and per field as the variable set defining each country/territory. 
We have also carried out cluster analysis, now using the number of citations per paper in each area. Since this indicator leads to smaller differences between countries, it is difficult for average linkage to produce separated clusters. Ward's method has shown to be more effective in these cases and the respective dendrogram is presented in Fig. 7. The patterns are not as well defined as those obtained resorting to the total number of citations. Geographical proximity is still visible as an underlying factor, but in some cases another nearest neighbour is selected.

\section{Conclusion}

The results presented in this work indicate that institutional dimension is a paramount factor in scientific ranking. In terms of policy, this supports the already stated idea that institutions should merge, to improve the respective placement in the rankings, especially in smaller countries.

Our conclusions further indicate that aspects related with geographical position are the dominant factors dictating both the relative importance of scientific areas in European countries and the time evolution of scientific productivity. Long term policies, planning and investment are still visible with clear patterns that contrast, for example, the Western Europe with the former Communist block countries. A corresponding degree of development in some countries also accounts for some of the groups. Direct cooperation between countries seems, unexpectedly, not sufficient to explain the structure obtained.

It is also recognised that a similar pattern is extracted using only the scientific and technological areas, as an evidence of the continuity existing between the sciences of nature and the humanities.

We have stressed that the underlying geographical factor is paramount for establishing the similarity patterns described in the present work. However, since this study is based on a complex 22 variable profile that determines the formation of the groups, the actual differences and similarities clearly result from a variety of factors. In fact, those specifically behind the development of, e.g., the area of Agricultural Sciences are probably independent from those governing Clinical Medicine or Chemistry. Some main aspects may be indicated, but it is difficult to identify all the factors behind a structure obtained from the whole set of areas. We believe that the patterns outlined in this work should be, in future analyses, contrasted with others obtained from social, economic or historical indicators.

\section{References}

Abdi, H. (2003). Factor rotations. In M. Lewis-Beck, A. Bryman, \& T. Futing (Eds.), Encyclopedia for research methods for the social sciences. CA: Thousand Oaks. Adam, D. (2002). The counting house. Nature, 415(2002), 726-729.

Almeida, J. A., Barbosa, S., Pais, L. M. S., \& Formosinho, A. A. C. C. S. J. (2007). Improving hierarchical cluster analysis: A new method with outlier detection and automatic clustering. Chemometrics and Intelligent Laboratory Systems, 87, 208-217.

Andras, P., \& Charlton, B. G. (2004). European science must embrace modernization. Nature, 429, 699.

Bishop, N., Gillet, V. J., Holliday, J. D., \& Willet, P. (2003). Chemoinformatics research at the University of Sheffield: A history and citation analysis. Journal of Information Science, 29, 249-267.

Braun, T. (1999). Bibliometric indicators for the evaluation of universities-intelligence from the quantitation of the scientific literature. Scientometrics, 45 , 425-432.

Braun, T., Dióspatonyi, I., Zádor, E., \& Zsindely, S. (2007). Journal gatekeepers indicator-based top universities of the world, of Europe and of 29 countries. A pilot study. Scientometrics, 71, 155-178.

Brereton, R. G. (2003). Chemometrics: Data analysis for the laboratory and chemical plant. Chichester: Wiley.

Coccia, M. (2008). Measuring scientific performance of public research units for strategic change. Journal of Informetrics, 2, 183-194.

Cronin, B. (2001). Bibliometrics and beyond: Some thoughts on web-based citation analysis. Journal of Information Science, 27, 1-7.

Csajbók, E., Berhidi, A., Vasas, L., \& Schubert, A. (2007). Hirsch-index for countries based on Essential Science Indicators data. Scientometrics, 73, 91-117.

Essential Science Indicators, The Thomson Corporation. Available at: http://scientific.thomson.com/products/esi (accessed November 2007); http://scientific.thomson.com/support/recorded-training/esi/ (accessed March 2008)

Feller, I., \& Gamota, G. (2007). Science indicators as reliable evidence. Minerva, 45, 17-30.

Garfield, E. (1955). Citation indexes for science: A new dimension in documentation through association of ideas. Science, 122, 108-111.

Glänzel, W. (2007). Characteristic scores and scales. A bibliometric analysis of subject characteristics based on long-term citation observation. Journal of Informetrics, 1, 92-102.

HDI, Human Development Indices: A statistical update 2008. Available at: http://hdr.undp.org/en/statistics (accessed December 2008). These data correspond to the year 2006 .

Hirsch, J. E. (2005). An index to quantify an individual's scientific research output. Proceedings of the National Academy of Sciences of the United States of America, 102, 16572-16596.

Horta, H., \& Veloso, F. M. (2007). Opening the box: Comparing EU and US scientific output by scientific field. Technological Forecasting and Social Change, 74, $1334-1356$.

International Monetary Fund, World Economic Outlook Database. Available at: http://imf.org/external/pubs/ft/weo/2007/oi/index.htm (accessed November 2007).

ISI Web of Knowledge, The Thomson Corporation. Available at: http://isiwebofknowledge.com/(accessed November 2007).

King, D. A. (2004). The scientific impact of nations. Nature, 430, 311-316.

Kostoff, R. N., Del Río, J. A., Cortés, H. D., Smith, C., Smith, A., Wagner, C., Leyddesdorff, L., Karypis, G., Malpohl, G., \& Tshiteya, R. (2007). Clustering methodologies for identifying country core competencies. Journal of Information Science, 33, 21-40.

Kozlowski, J., Radosevic, S., \& Ircha, D. (1999). History matters: The inherited disciplinary structure of the post-communist science in countries of central and eastern Europe and its restructuring. Scientometrics, 45, 137-166.

Leydesdorff, L. (2005). Evaluation of research and evolution of science indicators. Current Science, 89, 1510-1517.

May, R. M. (1997). The Scientific wealth of nations. Science, 275, 793-796.

May, R. M. (1998). The scientific investments of Nations. Science, 281, 49-51.

Moed, H. F., De Bruin, R. E., \& van Leeuwen, Th. N. (1995). New bibliometric tools for the assessment of national research performance: Database description, overview of indicators and first applications. Scientometrics, 33, 381-422.

Ortega, J. L., \& Aguillo, I. F. (2008). Linking patterns in European Union countries: Geographical maps of the European academic web space. Journal of Information Science, 34, 705-714.

Polanyi, M. (1983). Personal knowledge. Towards a post-critical philosophy. London: Routledge and Kegan Paul., p. 266 [paperback edition 1983] 
Schreiber, M. (2008). A modification of the $h$-index: The $h_{\mathrm{m}}$-index accounts for multi-authored manuscripts. Journal of Informetrics, 2, 211-216.

Schubert, A., \& Glänzel, W. (2006). Cross-national preference in co-authorship, references and citations. Scientometrics, 69, 409-428.

Snow, C. P. (1964). The two cultures and a second look. An expanded version of the two cultures and the scientific revolution. Cambridge: Cambridge UP.

Thelwall, M. (2008). Bibliometrics to webometrics. Journal of Information Science, 34, 605-621.

Tian, Y., Wen, C., \& Hong, S. (2008). Global scientific production on GIS research by bibliometric analysis from 1997 to 2006. Journal of Informetrics, 2, 65-74.

van Raan, A. F. J. (1998). In matters of quantitative studies of science the fault of theorists is offering too little and asking too much. Scientometrics, 43 ,

129-139.

van Raan, A. F. J. (2005). Fatal attraction: Conceptual and methodological problems in the ranking of universities by bibliometric methods. Scientometrics, $62,133-143$.

Web of Science ${ }^{\circledR}$. The Thomson Corporation. Available at: http://scientific.thomson.com/support/products/wos7/ (accessed November 2007).

Weingart, P. (2005). Impact of bibliometrics upon the science system: Inadvertent consequences? Scientometrics, 62, 117-131.

Willett, P. (1988). Recent trends in hierarchical document clustering: A critical review. Information Processing \& Management, 24, 577-597.

Winkler, P. (2007). Eminence of scientists in the light of the $h$-index and other scientometric indicators. Journal of Information Science, 33, 481-491. 